\title{
Análise descritiva dos trabalhos de conclusão de curso da Faculdade de Odontologia, UFPel
}

\author{
Luiz Alexandre Chisini*; Heloisa Grehs e Silva**; Kaio Heide Sampaio Nóbrega**; Marcus Cristian \\ Muniz Conde***; Marcos Britto Corrêa ${ }^{* * * *}$; Flávio Fernando Demarco ${ }^{* * * *}$
}

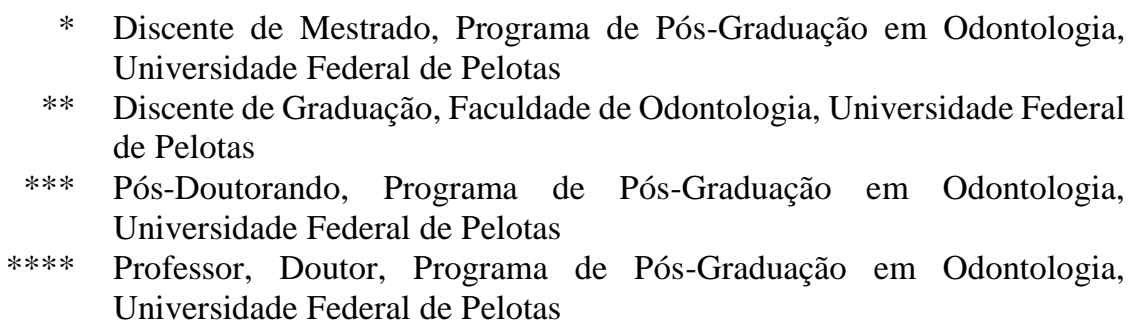

Recebido em 09/10/2016. Aprovado em 10/12/2016.

\begin{abstract}
RESUMO
Os Trabalhos de Conclusão de Curso (TCCs) se tornaram obrigatórios na Faculdade de Odontologia da Universidade Federal de Pelotas (FO-UFPel) a partir de 2006, sendo requisito indispensável para a obtenção do título de Cirurgião-Dentista. A compreensão de como os TCCs estão sendo realizados é imprescindível para um correto direcionamento de estratégias na construção dos currículos de graduação, uma vez que até o momento poucos estudos foram conduzidos com a finalidade de analisar os TCCs realizados nos cursos de Odontologia do Brasil. Desta forma, o objetivo deste estudo foi realizar um levantamento dos dados referentes aos TCCs realizados na FO-UFPel até 2014. As variáveis investigadas foram área de concentração do trabalho e tipos de estudos/metodologia empregada. Além disto foi realizada uma busca nas bases de dados PubMed, SciELO e Google Acadêmico para investigar se o trabalho foi publicado em periódicos científicos e o respectivo Qualis. Os dados foram tabulados e analisados descritivamente. Foram encontrados 474 TCCs, (impressos e em formato digital) sendo que a maioria deles foi realizada na área de Dentística $(11,4 \%)$, Saúde Coletiva $(11,0 \%)$ e Cirurgia Oral (10,5\%). Do total de TCCs, 25,7\% foram revisões narrativas da literatura. Apenas $9 \%$ das monografias foram publicadas em periódicos científicos. Portanto, é possível concluir que a maioria dos TCCs realizados na FO-UFPel são revisões narrativas da literatura e que a taxa de publicação destes trabalhos é pequena. Além disso, recomenda-se que novas estratégias sejam empregadas pelos gestores da universidade para propiciar que os TCCs possam ser referência na difusão do conhecimento à comunidade acadêmica.

Descritores: Educação em Odontologia. Metodologia. Avaliação. Odontologia. Currículo.
\end{abstract}

\section{INTRODUÇÃO}

A integralização de atividades de pesquisa conjuntamente com atividades de ensino e extensão são requisitos indispensáveis para uma formação acadêmica completa ${ }^{1-3}$. Estas atividades devem ser pensadas coletivamente para integrar os processos ensino /aprendizagem $^{4,5}$ e estar incluídas nos projetos políticos pedagógicos dos cursos de ensino superior como estratégias de consolidação e 
aprofundamento profissional ${ }^{6-8}$. As Diretrizes Curriculares Nacionais para os cursos de graduação em Odontologia instituíram que o estudante, ao final do curso, deve compreender e saber intervir no processo saúde/doença de forma individual e coletiva ${ }^{1,9}$. Desta forma, é indispensável que o egresso apresente uma base científica sólida e consiga interpretar e aplicar os diversos conhecimentos científicos disponíveis na literatura ${ }^{10}$.

Assim, a realização do trabalho de conclusão de curso (TCC), também conhecido como monografia, passou a ser um requisito indispensável para a obtenção do título de cirurgião-dentista visando maior vivência do futuro profissional com o método científico propriamente dito ${ }^{1}$. Além disto, a utilização dos TCCs como forma de avaliação final dos acadêmicos é amplamente utilizada pelos cursos de ensino superior no Brasil. A elaboração do TCCs deve ser fortemente alicerceada na utilização do método científico como ferramenta na construção de um documento final. Ele deve ser realizado nas normas da Associação Brasileira de Normas Técnicas (ABNT) ou nas normas internas das universidades, sob orientação de um docente ${ }^{11}$. Além disto, o TCC é apresentado a uma banca formada por profissionais, previamente convidados, os quais, por sua vez, avaliam os preceitos técnicos e metodológicos do trabalho e o classificam como satisfatório ou não para a obtenção do título de graduado.

Estes trabalhos acadêmicos podem servir como ferramentas institucionais importantes para o desenvolvimento crítico/reflexivo dos futuros profissionais de saúde ${ }^{6}$. Um estudo desenvolvido na universidade de Standford (EUA) observou que incentivos financeiros e profissionais no desenvolvimento de atividades de pesquisa em conjunto com alunos de graduação podem proporcionar uma oportunidade educacional positiva, além disso, gerar desenvolvimento científico com publicação de artigos e resumos de congressos por parte dos acadêmicos ${ }^{12}$. Na Faculdade de medicina da Universidade Federal da Bahia foi observado que o contato com a pesquisa, seja na iniciação científica ou durante o TCC, foi tido como positivo no desenvolvimento profissional e pessoal. $\mathrm{O}$ discurso coletivo construído com base nas entrevistas individuais indicaram que os estudantes reconhecem o valor da pesquisa científica curricular, cujas atividades se encerram com a elaboração e apresentação dos TCCs ${ }^{13}$.

Tendo em vista que o currículo acadêmico e o projeto político-didático-pedagógico da Faculdade de Odontologia da Universidade Federal de Pelotas (FO-UFPel) estão em processo de reestruturação, a compreensão de como os TCCs estão sendo realizados é imprescindível para o correto direcionamento de estratégias na construção de um novo currículo. Ademais, até o presente momento poucos estudos foram conduzidos com a finalidade de analisar os TCCs dos cursos de Odontologia no Brasil. Assim, o objetivo do presente estudo foi realizar um levantamento dos dados referentes aos TCCs para observar os tipos mais prevalentes de estudos realizados na FO-UFPel, assim como investigar se os mesmos estão sendo publicados em periódicos científicos.

\section{METODOLOGIA}

Foi realizado um estudo transversal retrospectivo com coleta de dados dos TCCs elaborados na FO-UFPel até 2014. Trabalhos anteriores a 2006 foram consultados diretamente na biblioteca da instituição, por estarem indexados apenas em formato impresso. A partir de 2006, os TCCs foram obtidos por consulta no sistema de gerenciamento do acervo das bibliotecas da UFPel. As variáveis de interesse investigadas foram área de concentração do trabalho, metodologia empregada e se o trabalho foi publicado em periódicos científicos. 
A área de concentração do trabalho foi coletada de acordo com a área indexada do trabalho (Dentística, Saúde Coletiva, Cirurgia Oral, Endodontia, Periodontia, Ortodontia, Odontopediatria, Patologia, Epidemiologia, Materiais Dentários, Prótese e Implantodontia). Quando os trabalhos estavam indexados em duas áreas de concentração, a área predominante do trabalho foi considerada. Trabalhos realizados em ciências básicas foram categorizados de acordo com a área odontológica específica e aqueles que não puderam ser agrupados nestas áreas foram categorizados em outros.

Os tipos de estudos foram coletados e agrupados como revisões sistemáticas, revisão narrativa da literatura, estudos transversais (qualitativos, quantitativos, observacionais, descritivos/analíticos observacionais), estudos de caso (relato de caso clínico e série de casos), estudos laboratoriais (in vitro, in vivo, in situ, exvivo), estudos clínicos (controlado, randomizado, não randomizado, intervencional), estudos longitudinais (caso-controle, observacional, retrospectivo) e revisões sistemáticas. Para averiguar se as monografias haviam sido publicadas em periódicos científicos, todos os TCCs encontrados foram pesquisados pelo título nas bases de dados SciELO, PubMed e Google Acadêmico.
Além disto, foi feita uma pesquisa adicional no currículo Lattes (http://lattes. cnpq.br/) do acadêmico e do professor orientador, com finalidade de averiguar se o TCC havia sofrido alteração no título previamente à sua publicação. Após isso, foi pesquisado o Qualis dos periódicos (https://sucupira.capes. gov.br/sucupira/public/consultas/coleta/veiculoP ublicacaoQualis/listaConsultaGeralPeriodicos.js f) nos quais as monografias haviam sido publicadas.

Os dados foram tabulados pelo software Microsoft Excel 2010@ e analisados descritivamente. O levantamento foi realizado durante o período de maio a junho de 2015.

\section{RESULTADOS}

Foram identificados quatrocentos e setenta e quatro (474) TCCs, dos quais a primeira indexação data de 2003. Além disto, observou-se elevação no número de registros a partir do ano de 2008, sendo o ano de 2014 com o máximo de indexações $(n=73)$. A distribuição dos TCCs por área de concentração foi de forma geral homogênea, com maiores números de monografias defendidas na área de Dentística $(11,4 \%)$, Saúde Coletiva $(11,0 \%)$ e Cirurgia Oral $(10,5 \%)$ (gráfico 1).

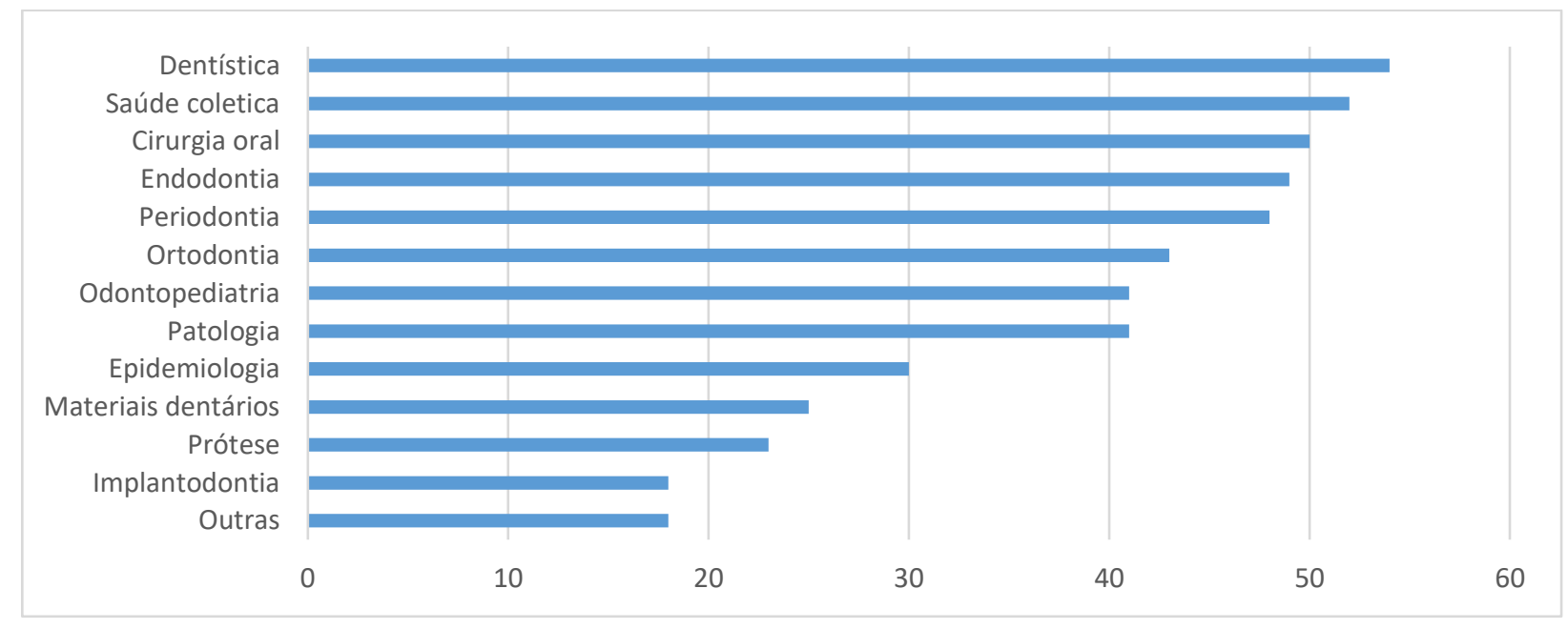

Gráfico 1. Distribuição dos TCCs realizados na FO-UFPel por área específica da Odontologia durante o período de 2003 a $2014(\mathrm{n}=474)$ 
No que diz respeito à metodologia elegida nos trabalhos, a revisão narrativa da literatura foi a mais utilizada, representando aproximadamente $25,7 \%$ dos TCCs. Estudos transversais constituem $23,6 \%$ dos trabalhos, seguidos por estudos de caso (relato de caso e série de casos) (gráfico 2). Em contrapartida, revisões sistemáticas da literatura, estudos longitudinais e estudos clínicos (randomizado, não randomizado, intervencional) foram os menos frequentes. Estudos utilizando metodologias in vivo (em animais) representaram apenas $1,7 \%$ dos TCCs.

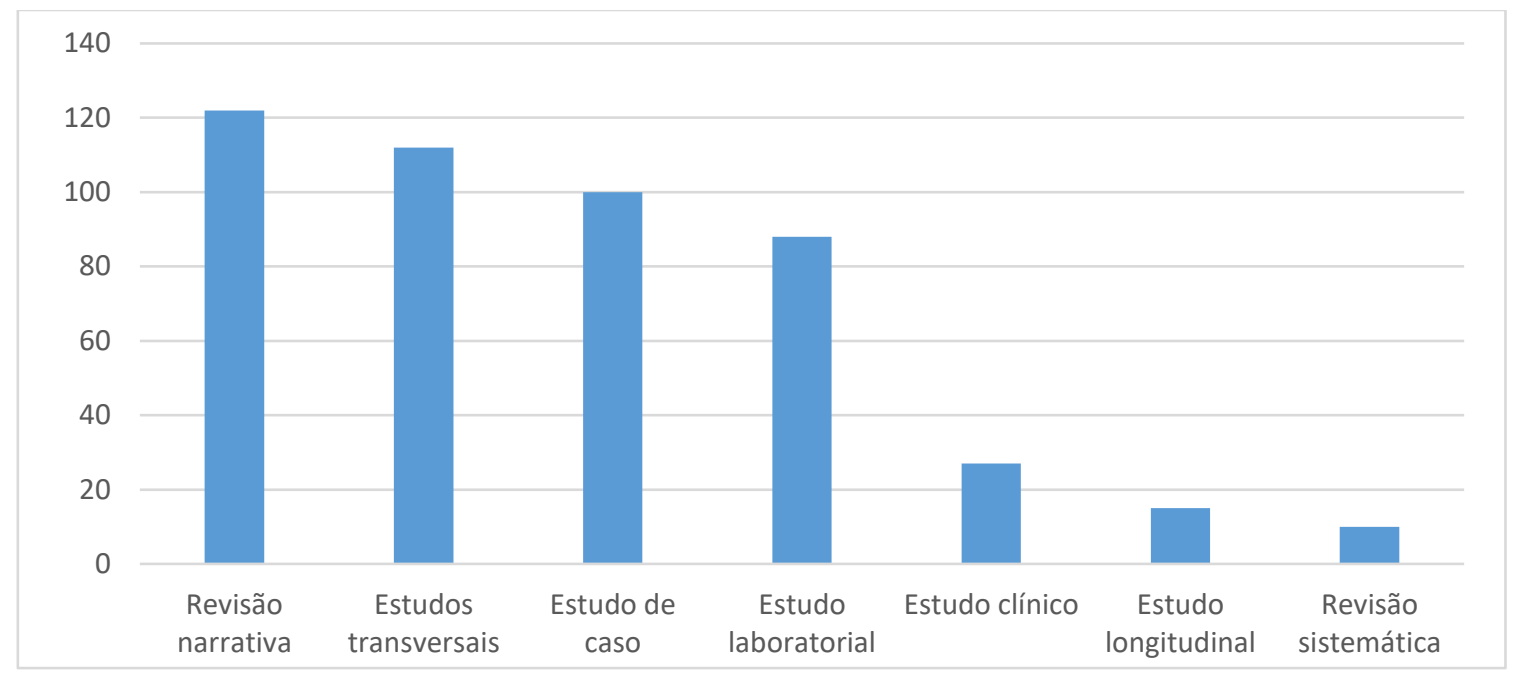

Gráfico 2. Desenhos de estudo utilizados na realização dos TCCs da FO-UFPel (n=474)

Além disso, observou-se que apenas 9\% dos trabalhos foram publicados em revistas científicas. Destes, a maioria $(31,6 \%)$ foi publicada em revistas de Qualis B4. No entanto, cinco TCCs foram publicados em revistas de Qualis A1 e quatro em revistas A2 (gráfico 3), revelando também um alto grau de impacto de pesquisas desenvolvidas por acadêmicos.

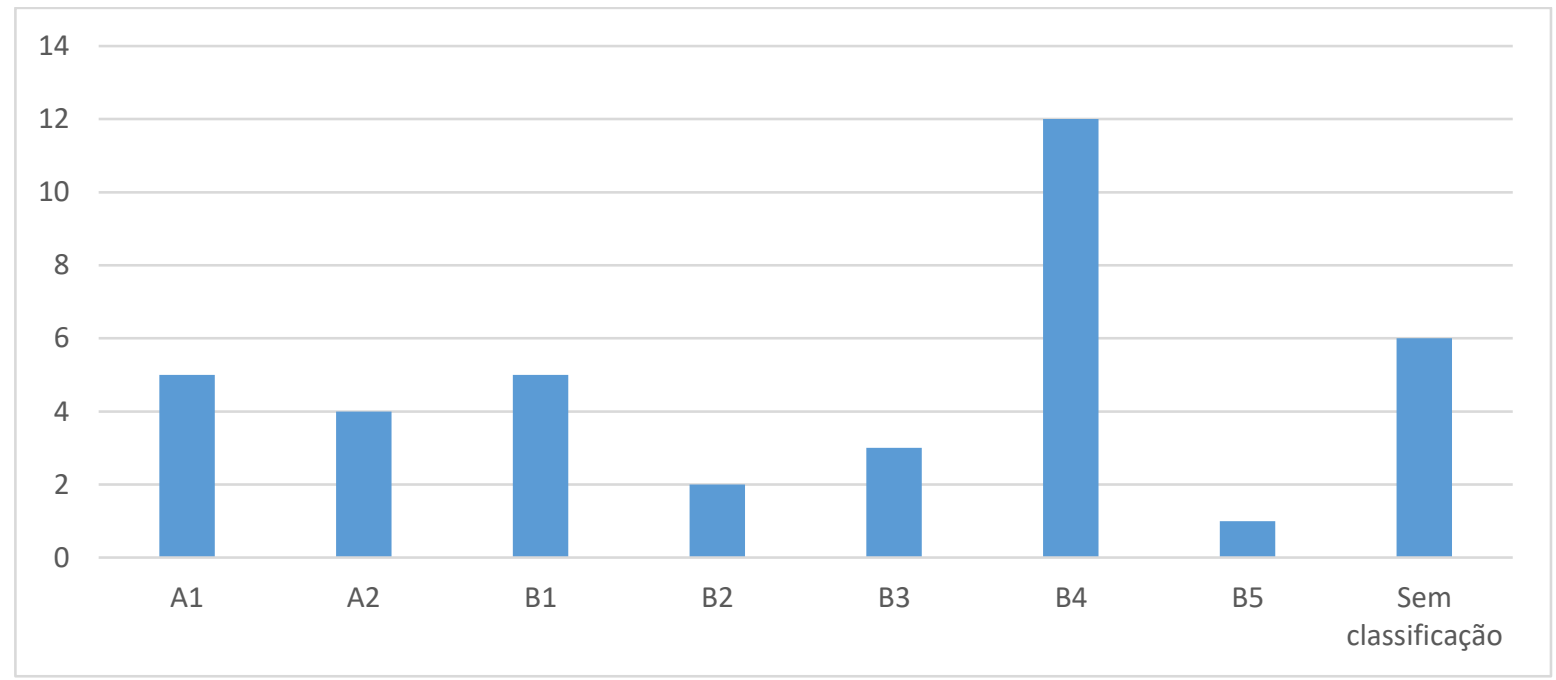

Gráfico 3. Conceito Qualis das revistas científicas nas quais os TCCs foram publicados $(\mathrm{n}=38)$ 


\section{DISCUSSÃO}

A participação em atividades extracurriculares, como a iniciação científica, durante a graduação é um fator que contribui para a formação de um bom profissional e deve ser apreciada e disponibilizada pelas universidades a fim de proporcionar um ambiente acadêmico que favoreça a formação de um profissional integral e crítico ${ }^{3,14,15}$. A realização do TCC permite que o aluno de graduação tenha contato ativo com as metodologias de pesquisa, proporcionando assim o desenvolvimento da capacidade crítica e questionadora ${ }^{6}$. No entanto, pouco se sabe sobre a perfil dos TCCs conduzidos nos cursos de odontologia no Brasil, da mesma forma como o possível impacto destas pesquisas em âmbito nacional e internacional.

Os TCCs se tornaram obrigatórios na FOUFPel a partir de 2006, visando contemplar as diretrizes curriculares nacionais. $\mathrm{O}$ mesmo começa a ser planejado no $8^{\circ}$ semestre com a entrega do nome do orientador ao colegiado de curso. Neste mesmo período realiza-se o projeto de pesquisa, o qual é posteriormente desenvolvido pelo acadêmico. No décimo e último semestre, o TCC deve ser redigido e entregue à banca avaliadora, sendo que sua defesa é requisito indispensável para a obtenção do título de cirurgião-dentista. O presente estudo identificou 474 TCCs apresentados durante o período de 2003 a 2014, com aumento no número de monografias a partir do ano de 2008. Isto pode ser explicado devido ao fato da universidade ter modificado a forma de ingresso, aderindo ao Sistema de Seleção Unificada (SISU) do Ministério da Educação, assim como ao Programa Nacional de Reestruturação e Expansão das Universidades Federais $(\mathrm{REUNI})^{16}$, o que proporcionou um incremento significativo no número de ingressos no curso (de 45 para 66).

Além disto, observou-se que duas das três áreas do conhecimento com maior número de trabalhos defendidos são aquelas em que a instituição oferece programas de pós-graduação. Isso pode ser devido ao fato da interação entre graduação e pós-graduação, que deve ser articulada e trabalhar indissociadamente na formação de acadêmicos e futuros professores. Alunos de stricto sensu (mestrado e doutorado) realizam estágio docência com acadêmicos de graduação, além de terem a possibilidade de atuar como coorientadores dos TCCs. Resultados semelhantes foram observados em um estudo recente que analisou os projetos de TCCs do curso de Odontologia da Universidade Federal do Rio Grande do Norte $^{10}$. O maior número de projetos de TCCs realizados foi nas áreas de Patologia Oral e Saúde Coletiva, áreas as quais apresentam programas de stricto sensu ${ }^{10}$. Desta forma, observa-se grande influência dos programas de pós-graduação sobre a pesquisa na graduação.

Revisões narrativas da literatura e estudos transversais foram os tipos de estudos mais utilizados, representando quase $50 \%$ dos trabalhos. Resultados diferentes foram observados na análise dos TCCs do curso de Odontologia da Escola Bahiana de Medicina e Saúde Pública, onde trabalhos de pesquisa foram os mais predominantes (36\%), seguidos por relatos de caso $^{17}$. No presente estudo, revisões sistemáticas e estudos longitudinais foram menos expressivos (2,1\% e 3,1\%, respectivamente). Da mesma forma, estudos utilizando modelos in vivo representaram menos de $2 \%$ das monografias. A pouca expressividade destes resultados pode ser explicada pela dificuldade de se conduzir tais metodologias, somada ao pouco tempo para o desenvolvimento e realização dos TCCs. Isto pode fazer com que os graduandos optem por metodologias com execução mais simplificada. Silva et al. ${ }^{11}$ (2009) observaram de forma semelhante que cerca de $87 \%$ dos trabalhos de conclusão de curso do Curso de Enfermagem da Universidade Estadual de Montes Claros foram 
estudos observacionais, enquanto que menos de $1 \%$ eram estudos experimentais. Estes achados ${ }^{11}$ corroboram com os dados do presente estudo que demonstram preferência dos estudantes por metodologias mais simples na condução da monografia. Além disto, deve-se ressaltar que o estudante necessita realizar o projeto, executá-lo e escrever o manuscrito em um curto período de tempo. Isto de fato dificulta a realização de estudos longitudinais, experimentais ou de intervenção. Desta forma, podemos sugerir que a realização do TCC seja conduzida durante um período prolongado da graduação, transformando assim o trabalho final do curso em uma constante trajetória de pesquisa. No entanto, mecanismos de incentivo e controle devem ser criados para tornar o processo homogêneo $\mathrm{e}$ constante ao longo do curso.

Um restrito espaço de tempo para o planejamento e execução do TCC pode ser fator sinérgico para resultar em um trabalho com pequeno impacto científico sem perspectiva de publicação. Neste estudo, observamos que cerca de $91 \%$ dos TCCs não foram encontrados em periódicos científicos. A publicação destes trabalhos é importante na medida em que amplia a difusão do conhecimento gerado. Além do papel de formar profissionais, a universidade é a principal responsável pelo crescimento científico/tecnológico do Brasil, apresentando papel de extrema importância. Apesar da baixa percentagem de publicações das monografias da FO-UFPel, deve-se ressaltar que diversos TCCs foram publicados em periódicos classificados como A1 e A2. Estes periódicos apresentam um elevado impacto na pesquisa em nível internacional, demonstrando que monografias conduzidas por acadêmicos podem prover contribuição substancial à pesquisa brasileira.

Assim, sugere-se o incentivo precoce dos estudantes em programas de Iniciação Científica, buscando estimular o envolvimento de novos estudantes na área da pesquisa, bem como recomenda-se que novas estratégias sejam empregadas pelos gestores da Universidade para propiciar que os TCCs possam ser referência na difusão do conhecimento à comunidade acadêmica.

\section{CONCLUSÃO}

A maioria dos TCCs realizados na FOUFPel é do tipo revisão narrativa e estudo transversal. Além disso, sua publicação em periódicos científicos é restrita, apesar de alguns terem sido publicados em periódicos de alto impacto científico.

\section{ABSTRACT \\ Descriptive analysis of work completion course of the graduate course in Faculty of Dentistry, UFPel}

The final paper (TCCs) became mandatory in the Faculty of Dentistry, Federal University of Pelotas (FO-UFPel) since 2006, and an indispensable requirement for obtaining DDS title. Understanding how the TCCs are being carried out is essential for proper targeting strategies in the construction of the undergraduate curriculum, since to date few studies were conducted in order to analyze the TCCs made in dentistry courses in Brazil. Thus, the aim of this study was to survey the data for TCCs performed in FO-UFPel by 2014. The variables were: area of work concentration, types of studies/methodology. In addition, a search was conducted in PubMed, SciELO and Google Scholar to investigate whether the work was published in scientific journals and their Qualis conceit. The data were tabulated and analyzed descriptively. 474 TCCs were founded (printed and digital format), and most of them were held in operative dentistry (11.4\%) followed by public health (11.0\%) and oral surgery (10.5\%). 25.7\% of TCCs were narrative reviews of the literature. Only $9 \%$ of monographs have been published in scientific journals. Therefore, we note that the majority of TCCs made in FO-UFPel are narrative literature reviews and the publication of these works is small. In addition, it is recommended that new strategies are employed 
by the university managers to provide that the TCCs can be reference in dissemination of knowledge to the academic community.

Descriptors: Education in Dentistry. Methodology. Evaluation. Dentistry. Curriculum.

\section{REFERÊNCIAS}

1. Brasil. Brasil. Conselho Nacional de Educação. Câmara de Educação superior. Resolução CNE/CES No 3, de 19 de fevereiro de 2002. Institui Diretrizes Curriculares Nacionais do Curso de Graduação em Odontologia. Brasil: CNE/CES. 2002. [Acesso em 09 out. 2016]. Disponível em: http://portal.mec.gov.br/cne/arquivos/pdf/CE S032002.pdf.

2. Grande I, Prochnow R, Saab R, Pizzatto E. Desafios na formação do Cirurgião-Dentista para o SUS. Rev ABENO. 2016;16(2):2-6. [Acesso em 03/12/2016]. Disponível em: https://revabeno.emnuvens.com.br/revabeno/ article/view/256.

3. Warmling A, Mello A, Naspolini D, Canto G, Souza E. Contribuições das atividades complementares na formação profissional em odontologia. Rev ABENO. 2012;12(2):190-7. [Acesso em 03/ dez. 2016]. Disponível em: http://revodonto.bvsalud.org/scielo.php?scrip $\mathrm{t}=$ sci_arttext\&pid=S16795954201200020000 9\&lng=es\&nrm=iso\&tlng=pt.

4. Júnior A, Miranda N, Assunção R, Silva S, Oliveira F, Oliveira R. Percepção de estudantes de Odontologia sobre metodologias ativas no processo de ensinoaprendizagem. Rev ABENO. 2016;16(3):66677. [Acesso em 03 dez. 2016]. Disponível em: https://revabeno.emnuvens.com.br/revabeno/ article/view/272/248.

5. Farias C, Cardoso B, Neto E, Carvalho R, Curtis D. Feedback no processo de aprendizagem: percepção dos estudantes de Odontologia em uma universidade brasileira. Rev ABENO. 2015;15(3):35-42. [Acesso em 03 dez. 2016]. Disponível em: https:// revabeno.emnuvens.com.br/revabeno/article/ view/160.

6. Saupe R, Wendhausen ALP, Machado HB. Modelo para a implantação ou revitalização de trabalhos de conclusão de curso. Rev Latinoam Enferm. 2004;12(1):09-14. [Acesso em 09/10/2016]. Disponível em: http://www. scielo.br/scielo.php? script $=$ sci_arttext\&pid $=$ S0104-11692004000100015.

7. Faé J, Junior M, Carvalho R, Esposti C, Pacheco K. A integração ensino-serviço em Odontologia no Brasil. Rev ABENO. 2016;16(3):7-18. [Acesso em 03 dez. 2016]. Disponível em: https://revabeno.emnuvens. com.br/revabeno/article/view/286.

8. Ferraz M, Falcão C, Pereira R, Pinheiro C, Nascimento E, Moura M. Projeto pedagógico do Curso de Odontologia: conhecimento discente. Rev ABENO. 2015;15(2):47-55. [Acesso em 03 dez. 2016]. Disponível em: https://revabeno.emnuvens.com.br/revabeno/ article/view/176.

9. Toassi R, Souza J, Baumgarten A, Rösing C. Avaliação curricular na educação superior em odontologia: discutindo as mudanças curriculares na formação em saúde no Brasil. Rev ABENO. 2012;12(2):170-7. [Acesso em 03 dez. 2016]. Disponível em: https:// revabeno.emnuvens.com.br/revabeno/article/ view/120.

10. Leite $\mathrm{B}$, Menêzes $\mathrm{T}$, Noro L. Análise bibliométrica de Trabalhos de Conclusão de um Curso de Odontologia no Nordeste brasileiro. Rev ABENO. 2015;15(3):16-25. [Acesso em 03 dez. 2016]. Disponível em: https://revabeno.emnuvens.com.br/revabeno/ article/view/172.

11. Silva V, Holzmann APF, Versiani CC, Figueiredo MFS, Lima ACAS, Vieira MA, et al. Análise dos trabalhos de conclusão de curso da graduação em enfermagem da UNIMONTES. Rev Eletrônica Enferm. 2009;11(1):133-43. [Acesso em 09 out. 2016]. Disponível em: https://www.fen.ufg.br/fen revista/v11/n1/pdf/v11n1a17.pdf.

12. Jacobs CD, Cross PC. The value of medical student research: the experience at Stanford University School of Medicine. Med Educ. 1995;29(5):342-6.

13. Guedes HTV, Guedes JC. Avaliação, pelos Estudantes, da Atividade "Trabalho de Conclusão de Curso" como Integralização do Eixo Curricular de Iniciação à Pesquisa Científica em um Curso de Medicina. Rev Bras Educ Med. 2012;36(2):162-71. [Acesso em 03 dez. 2016]. Disponível em: http:// 
wwW.scielo.br/scielo.php?script=sci_arttext \&pid=S0100-55022012000400003.

14. Cunha MR, Fonsêca GS, de Araujo ME, Zilboviciu C. A Iniciação Científica como estratégia pedagógica para integrar ensino e serviço no SUS. Rev ABENO. 2016;16(2):3344. [Acesso em 09 out. 2016]. Disponível em: https://revabeno.emnuvens.com.br/revabeno/ article/view/250.

15. Farias C, Carvalho R, Laiber L, Belotti L, Pacheco K. Pensamento crítico e a formação de profissionais em Odontologia: uma revisão narrativa da literatura. Rev ABENO. 2016;16(1):73-87. [Acesso em [Acesso em 03 dez. 2016]. Disponível em: https://revabeno. emnuvens.com.br/revabeno/article/view/233.

16. Marques ACH, Cepêda VA. Um perfil sobre a expansão do ensino superior recente no Brasil: aspectos democráticos e inclusivos.
Perspectivas. 2012;42:161-92. [Acesso em 09 out. 2016]. Disponível em: http://seer. fclar.unesp.br/perspectivas/article/view/5944. 17. Costa R, Vieira R, Cavalcanti A, Tunes U, Ribeiro E, Oliveir V. Perfil da pesquisa acadêmica odontológica da EBMSP. Rev ABENO. 2015;15(70-76). [Acesso em 03 dez. 2016]. Disponível em: https://revabeno. emnuvens.com.br/revabeno/article/view/145.

Correspondência para:

Luiz Alexandre Chisini

e-mail: alexandrechisini@ hotmail.com

Universidade Federal de Pelotas

Faculdade de Odontologia

Rua Gonçalves Chaves 457, sala 501

96015-560 Pelotas/RS 